\title{
CUIDADO AO IDOSO EM DISTANCIAMENTO SOCIAL DURANTE A PANDEMIA: PREVENÇÃO DO TROMBOEMBOLISMO VENOSO
}

\section{Milara Barp'}

ORCID:0000-0002-7326-2189

Suelen Gomes Malaquias'

ORCID:0000-0001-8530-9100

Cynthia Assis de Barros Nunes' ORCID:0000-0001-7019-7468

Ana Railka de Souza Oliveira-Kumakura" ORCID: 0000-0002-7075-7987

Marina Aleixo Diniz Rezende ${ }^{\text {III }}$ ORCID: 0000-0003-3069-9185

Valéria Pagotto'

ORCID: 0000-0002-5590-2453

' Universidade Federal de Goiás. Goiânia, Goiás, Brasil.

" Universidade Estadual de Campinas. Campinas, São Paulo, Brasil.

III Pontificia Universidade Católica de Goiás. Goiânia, Goiás, Brasil.

Autor Correspondente: Milara Barp E-mail:enfmi.barp@gmail.com

Como citar:

Barp M, Malaquias SG, Nunes CAB, Kumakura ARSO, Rezende MAD, Pagotto V. Cuidado ao idoso em distanciamento social durante a pandemia: prevenção do tromboembolismo venoso. In: Santana

RF (Org.). Enfermagem gerontologica no cuidado do idoso em tempos da COVID 19.

2.ed.rev. Brasilia, DF: Editora ABen; 2020. p 122-130. (Serie Enfermagem e Pandemias, 2). https://doi.org/10.51234/aben.20.e02.c19

\section{INTRODUÇÃO}

O distanciamento social é uma das primeiras, e principais recomendações da Organização Mundial de Saúde (OMS) para enfrentamento da pandemia. Embora proporcione a prevenção da COVID-19 entre os idosos, o distanciamento social pode trazer algumas repercussões como comprometimento de atividades cotidianas, dos hábitos regulares de vida, das relações familiares e dos papéis sociais o que facilita o desencadeamento, agravamento ou recidiva de doenças e outros agravos à saúde ${ }^{1-5}$.

O tromboembolismo venoso (TEV) pode ter sua incidência aumentada durante a pandemia, uma vez que os seus fatores de risco são convergentes com algumas repercussões do distanciamento social. Sendo assim, fatores como redução de atividades físicas em casa, preferência por atividades com menor gasto de energia, imobilidade, descontinuidade de cuidados com à saúde e diminuição ou ausência de cuidados preventivos no domicílio podem repercutir no autocuidado, cuidado e necessidade de cuidados dos idosos, aumentando portanto, o risco de TEV 5 .

Considerando que os eventos tromboembólicos nos idosos são desencadeados a partir da interação de uma variedade de fatores de risco, esses podem ser agravados frente à recomendação atual de distanciamento social, é importante refletir sobre a ocorrência de TEV em idosos, sobre a relação dos seus fatores de risco com o distanciamento social, bem como sobre as ações da equipe de enfermagem para o autocuidado nesse grupo.

\section{OBJETIVO}

Refletir sobre as repercussões do distanciamento social na ocorrência de eventos tromboembólicos frente à pandemia da COVID-19, e sugerir cuidados de enfermagem para prevenção desses eventos. 


\section{MÉTODO}

Trata-se de estudo de reflexão teórica, desenvolvido com aporte na literatura, bem como na teoria do Autocuidado de Dorothea Orem. A apresentação foi organizada em duas categorias, a saber: eventos tromboembólicos venosos em idosos: ocorrência e fatores de risco; e prevenção de eventos tromboembólicos em idosos em distanciamento social à luz da Teoria Geral do Autocuidado de Dorothea Orem.

\section{RESULTADOS}

\section{Eventos tromboembólicos venosos em idosos: ocorrência e fatores de risco}

O TEV caracteriza-se pelo desenvolvimento de trombos no sistema venoso, que são manifestados pela trombose venosa profunda (TVP) e tromboembolismo pulmonar (TEP). São ocasionados pela associação de fatores que geram um desequilíbrio entre componentes anti e pró-trombóticos no organismo, formando coágulos patológicos. A fisiopatologia envolve os três fatores classicamente descritos pela "tríade de Virchow": lesão endotelial, lentificação do fluxo sanguíneo e aumento na coagulabilidade do sangue ${ }^{7}$.

À medida que a idade avança, o TEV torna-se mais frequente. Com o envelhecimento há alterações no sistema vascular e de coagulação sanguínea, que somadas às condições comuns em idosos como as múltiplas morbidades, hospitalizações, diminuição da mobilidade e das atividades cotidianas favorecem os eventos tromboembólicos venosos ${ }^{8}$.

A ocorrência dos eventos tromboembólicos foi avaliada em estudos anteriores à pandemia em diferentes contextos de cuidado à idosos, como hospitais, instituições de longa permanência (lares de idosos) e domicílio ${ }^{9-11}$; e sua frequência varia a depender do tempo de acompanhamento, local do estudo e do perfil dos idosos.

No âmbito hospitalar, em uma coorte retrospectiva de dez anos, o TEV teve um incremento de 19,8\% ao ano, devido ao aumento de casos de embolia pulmonar, e das multimorbidades ${ }^{12}$. Em outro estudo de 30 meses de seguimento, a incidência acumulada foi de $14,8 \%$ após 14 anos, com alta proporção de recidivas ${ }^{13}$. Já em idosos hospitalizados com fratura de fêmur, a taxa de incidência de TEV foi de 18,9\% no período pré-operatório, sendo a Trombose Venosa Profunda (TVP) o evento mais comum ${ }^{14}$.

Em instituições de longa permanência, em idosos do Reino Unido moradores de 45 casas lares, a incidência foi de 0,71 a 2,48 por 100 pessoas-ano ${ }^{10}$. Em uma análise retrospectiva de 181 casas de repouso dos Estados Unidos, 3,7\% dos idosos tinham TEV na admissão, e durante a permanência a incidência foi de 3,68 casos por 100 pessoas-ano ${ }^{15}$.

Quanto aos estudos com idosos residentes em domicílios da comunidade, idosos frágeis apresentam maior suscetibilidade à TEV, sendo que em idosos norte-americanos a incidência foi de 2,7 por 1000 pessoas-ano nos pré-frágeis, aumentando para 4,1 entre os frágeis ${ }^{16}$. Em idosos usuários da atenção primária, a incidência verificada foi de 8,0 por 1.000 pessoas-ano ${ }^{11}$.

Além da idade avançada ${ }^{10}$, outros fatores contribuem para o TEV como polifarmácia ${ }^{15}$, distúrbios de movimento coexistentes ${ }^{14}$, baixo nível de atividade física ${ }^{15}$, e repouso no leito por mais de sete dias ${ }^{12}$. As multimorbidades tem destaque e aumentam o risco do TEV em todos os cenários de cuidados ${ }^{9,14}$. Doenças como hipertensão arterial, infecção pulmonar, câncer, diabetes e doença cardíaca coronariana representam fatores de risco para o desenvolvimento do TEV7,9,16. Além disso, a obesidade, tabagismo e o consumo excessivo de álcool, contribuem para o risco aumentado ${ }^{7}$. Em instituições de cuidado prolongado, são muito presentes fatores como imobilidade prolongada ${ }^{10}$, trombose venosa profunda (TVP) ${ }^{17}$, e mobilidade física reduzida relacionada a comprometimento cognitivo (demência) ${ }^{17}$.

Essa breve revisão sobre a ocorrência e fatores de risco para o TEV em idosos sinaliza que se trata de um evento comum no âmbito hospitalar e em instituições de longa permanência. Nesse sentido, 
independente do contexto em que os eventos tromboembólicos venosos são identificados e da sua frequência, no contexto de pandemia sua ocorrência pode ser aumentada nos diferentes cenários de cuidado, incluindo o ambiente domiciliar. Embora as pesquisas no âmbito domiciliar tenham sido incipientes comparado aos outros locais, durante a pandemia, idosos terão suas atividades reduzidas impactando em eventos tromboembólicos.

Apesar de ainda não estar totalmente esclarecido, observa-se que a infecção pelo vírus Sars-CoV-2, que ocasiona doença respiratória aguda grave, favorece o aumento dos distúrbios de coagulação ${ }^{18}$. Sendo assim, o distanciamento social é uma tentativa de proteger os idosos dessa enfermidade e suas complicações, os quais devem permanecer por tempo prolongado em seus domicílios.

Por outro lado, o distanciamento social, poderá acentuar os fatores envolvidos no TEV, uma vez que limita atividades funcionais do idoso e mobilidade física repercutindo em possíveis alterações na coagulação preexistentes ou não. Além disso, para idosos que já apresentam TVP, a descontinuidade do tratamento pela dificuldade de acesso aos serviços também poderá agravar quadros prévios. Assim, é importante identificar nos espaços de cuidado, os fatores de risco para TEV, no sentido de preveni-los ou monitorá-los frente à recomendação de distanciamento social.

\section{Prevenção de eventos tromboembólicos em idosos em distanciamento social à luz da Teoria Geral do Autocuidado de Dorothea Orem}

No atual panorama de pandemia da COVID-19, torna-se fundamental o papel do enfermeiro na prevenção dos eventos tromboembólicos no idoso. Sua atuação envolve a assistência direta em âmbito hospitalar, mas deve se estender ao ensino sobre os fatores de risco e medidas preventivas em contextos não hospitalares, como em idosos que permanecem restritos ao ambiente domiciliar ou de instituições de longa permanência para idosos (ILPI), em decorrência do período de distanciamento social.

Nesse sentido, a "agência de enfermagem"19-20 constitui-se uma ação deliberada para alcance do autocuidado dos idosos em distanciamento social, a partir da estruturação dos sistemas de enfermagem correspondentes. Sistemas de enfermagem, por sua vez, são designados para o alcance da saúde, superação de déficits de autocuidado e/ou promoção e preservação do autocuidado ${ }^{20}$, sendo no contexto discorrido, a prevenção de eventos tromboembólicos venosos observando a manutenção do autocuidado dos idosos.

Com vistas à organização da assistência, esses sistemas de enfermagem são categorizados em: apoio educativo, parcialmente compensatório e totalmente compensatório. Dessa forma, o enfermeiro planeja sua atuação de forma mais eficaz ${ }^{19-20}$.

Os "fatores condicionantes básicos de autocuidado" 20 , na perspectiva de cuidado integral do idoso em distanciamento social, devem ser atentamente considerados pelo enfermeiro pela tendência à generalização das recomendações, característica de contextos de pandemia como a da COVID19. Entre esses condicionantes destacam-se: fatores familiares, ambientais, socioculturais, hábitos de vida e adequação e disponibilidade de recurso para ocorrência de determinado requisito de autocuidado relacionado à prevenção de tromboembolismo venoso, como por exemplo, aquisição de meias compressivas.

Ressalta-se a necessidade de considerar os cuidadores dos idosos, em especial familiares, como agências de autocuidado e, portanto, foco de ações de enfermagem concomitantemente à agência de autocuidado do idoso, pela possibilidade de repercussão na própria assistência a este.

Esse raciocínio pode ser evidenciado no sistema de enfermagem de apoio educativo ${ }^{20}$, em que o idoso apresenta habilidades para atividades básicas e instrumentais de vida diária. Nesse caso, o enfermeiro deve avaliar fatores de risco para o TEV e apresentá-los ao idoso, familiar e/ou cuidador, sendo que nas dificuldades de engajamento entre esses indivíduos há comprometimento, consequentemente, no atendimento a esse requisito de autocuidado do idoso. 
Entre os fatores de risco estão as doenças crônicas, tais como, câncer, doença pulmonar obstrutiva crônica, doenças cardiovasculares e doenças inflamatórias agudas, pois propiciam distúrbios da coagulação. Obesidade, tabagismo e o consumo excessivo de álcool também contribuem para o risco aumentado de $\mathrm{TEV}^{7,16}$, assim como a alta hospitalar recente. Idosos nesta situação são mais propensos ao TEV, com aproximadamente $50 \%$ dos casos associados a um período de internação hospitalar prévio ou atual ${ }^{16}$.

No que se refere ao sistema parcialmente compensatório, pondera-se em direção aos casos de limitações de mobilidade do idoso e prejuízos da cognição, aos quais serão necessárias intervenções de enfermagem que equilibrem essas demandas e que podem ser exacerbadas a partir da necessidade de distanciamento no cenário domiciliar. Dessa feita, para o sistema totalmente compensatório, a enfermagem desempenhará atividades básicas e instrumentais de vida diária concomitantemente aos cuidados preventivos de eventos tromboembólicos, considerando algum nível de julgamento de autocuidado que esse idoso apresente. Algumas considerações clínicas que se destacam em relação ao TEV e que transitam nos três (3) sistemas de enfermagem, que serão destacadas a seguir.

A redução significativa da mobilidade em idosos, que pode estar relacionada a progressão de quadros demenciais, limitações ortopédicas, paresias ou parestesias, deve receber atenção especial, pois propicia a estase sanguínea e ocorrência do TEV 8 . Portanto, intervenções ativas e/ou passivas, que reduzem a imobilidade física, conforme a capacidade funcional do idoso, aumentam a aptidão física a qual reduz o risco de TEV pela redução da estase sanguínea ${ }^{21}$.

Além disso, o acometimento cognitivo em alguns idosos que pode estar associado ainda a uma diminuição da rede de apoio devido ao distanciamento social, pode gerar uma situação preocupante, como a descontinuidade dos cuidados com à saúde, principalmente em relação ao tratamento medicamentoso para doenças crônicas ou tromboprofiláticos, com consequente risco de eventos tromboembólicos.

Ainda considerando o idoso mais fragilizado em distanciamento social, esse precisa ser assistido visando garantir sua adequada hidratação, pois estudos apontam que o estado de desidratação associado a outros fatores, favorece a hipercoagulabilidade sanguínea ${ }^{10}$.

Outro ponto que deve ser considerado refere-se ao reconhecimento precoce de sinais e sintomas de TEV. A sintomatologia discreta e inespecífica dificulta o reconhecimento da doença em fase inicial, especialmente naqueles com prejuízos cognitivos e os afastados do cuidado contínuo, devido o contexto epidemiológico. A trombose venosa profunda se caracteriza por edema em membro unilateral, hiperemia ou palidez local, hipersensibilidade e aumento da temperatura no membro comprometido ${ }^{7}$. A promoção do ensino, ao idoso e cuidador, do reconhecimento do quadro clínico de TEV, configura-se em considerar o idoso, agência de autocuidado no âmbito do sistema apoio educativo.

No quadro 1, são descritos os principais cuidados de enfermagem aos idosos em domicílio, no contexto da pandemia de COVID19. Esses cuidados estão baseados na intervenção "Precauções contra embolia", da Classificação de Intervenções de Enfermagem ${ }^{22}$, bem como recomendações da literatura especializada, e estão categorizados a partir da Teoria Geral do Autocuidado ${ }^{19}$ e as taxonomias de diagnósticos de enfermagem NANDA-123 e CIPE ${ }^{24}$.

Ressalta-se que idosos pós-hospitalizados para tratamentos clínicos ou cirúrgicos e/ou com diversos fatores de risco para tromboembolismo venoso podem necessitar de tratamentos específicos para profilaxia, tais como, administração de medicações tromboprofiláticas, aplicações de intervenções mecânicas e físicas conforme prescritos, exigindo cuidados totais ou parciais de familiares e/ou cuidadores, que no contexto da pandemia não podem ser interrompidos.

Desse modo, faz-se imprescindível à sensibilização dos familiares e/ou cuidadores dos idosos na preservação do cuidado de saúde durante o período de distanciamento social. A enfermagem gerontológica deve atuar buscando possibilidades junto com os familiares, principalmente os mais fragilizados e com doenças crônicas, para promover medidas que evitem o risco de ocorrência de eventos tromboembólicos. 
Quadro 1 - Ações de enfermagem possíveis aos idosos no contexto da pandemia de COVID19 baseados na Classificação de Intervenções de Enfermagem (NIC, 2016) ${ }^{22}$ e literatura especializada, categorizados a partir da Teoria Geral do Autocuidado ${ }^{19}$ e taxonomias de diagnósticos de enfermagem NANDA-I (2018) ${ }^{23}$ e CIPE (2019) ${ }^{24}$.

\begin{tabular}{|c|c|c|c|c|}
\hline $\begin{array}{l}\text { Sistemas de } \\
\text { enfermagem }\end{array}$ & & $\begin{array}{l}\text { Requisitos de } \\
\text { autocuidado }\end{array}$ & $\begin{array}{l}\text { Diagnósticos de } \\
\text { enfermagem }\end{array}$ & Ações de enfermagem \\
\hline \multirow{9}{*}{ 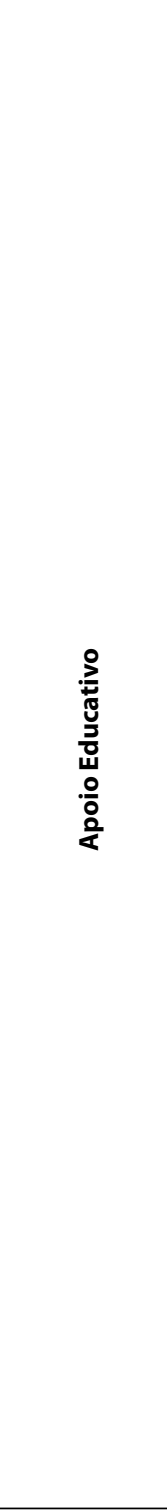 } & \multirow{9}{*}{ 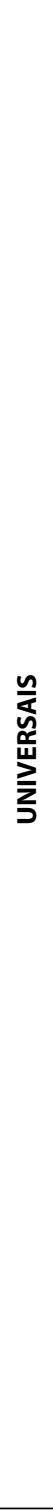 } & \multirow{2}{*}{$\begin{array}{l}\text { Manutenção de } \\
\text { ingestão hídrica }\end{array}$} & $\begin{array}{l}\text { Volume de líquidos } \\
\text { deficiente (NANDA-I 00027) }\end{array}$ & \multirow{2}{*}{$\begin{array}{l}\text { Orientar o idoso, familiares e/ou cuidadores quanto ao } \\
\text { consumo adequado de líquidos para garantir hidratação } \\
\text { corporal. }\end{array}$} \\
\hline & & & $\begin{array}{l}\text { Ingestão de líquidos } \\
\text { prejudicada (CIPE 10047330) }\end{array}$ & \\
\hline & & $\begin{array}{l}\text { Manutenção } \\
\text { de ingestão de } \\
\text { alimentos }\end{array}$ & $\begin{array}{l}\text { Conhecimento sobre Regime } \\
\text { Dietético prejudicado (CIPE } \\
\text { 10023772) } \\
\text { Conhecimento deficiente } \\
\text { (NANDA-I 00126) }\end{array}$ & $\begin{array}{l}\text { Orientar o idoso, familiar e/ou cuidador sobre a ingesta } \\
\text { de uma dieta balanceada de alimentos ricos em vitamina } \\
\mathrm{K} \text { (p. ex. vegetais de folhas verde-escuras). A dosagem do } \\
\text { medicamento anticoagulante deve ser ajustada ao consumo } \\
\text { alimentar desses alimentos, pois podem interferir com o uso de } \\
\text { tromboprofiláticos. }\end{array}$ \\
\hline & & \multirow{5}{*}{$\begin{array}{l}\text { Manutenção } \\
\text { de atividade e } \\
\text { repouso }\end{array}$} & \multirow{5}{*}{$\begin{array}{l}\text { Mobilidade física prejudicada } \\
\text { (NANDA-I 00085) } \\
\text { Risco de não Adesão ao } \\
\text { Regime de Exercício Físico } \\
\text { (CIPE 10022657) }\end{array}$} & $\begin{array}{l}\text { Ao considerar o contexto do domicilio do (a) idoso (a), encorajá- } \\
\text { lo (a) a realizar caminhadas, com aumento progressivo no } \\
\text { tempo de duração, em trajetos conhecidos de seu domicilio, } \\
\text { preferencialmente quintal ou áreas, e em que não haja móveis } \\
\text { e/ou desníveis. }\end{array}$ \\
\hline & & & & $\begin{array}{l}\text { Ensinar sobre exercícios de flexão e extensão de pés e pernas } \\
\text { pelo menos } 10 \text { vezes a cada hora e/ou programa de exercícios } \\
\text { para descongestão venosa } 3 \text { vezes por } \text { dia }^{25} \text {. }\end{array}$ \\
\hline & & & & $\begin{array}{l}\text { Orientar sobre a possibilidade de integração a grupos virtuais } \\
\text { de Dança Sênior }{ }^{\circledR} \text { e/ou verificar suporte de professor de } \\
\text { educação física para alongamentos e exercícios calistênicos. }\end{array}$ \\
\hline & & & & Desaconselhar massagear ou comprimir os músculos da perna. \\
\hline & & & & $\begin{array}{l}\text { Orientar para evitar manter as pernas cruzadas por muito } \\
\text { tempo. }\end{array}$ \\
\hline & & $\begin{array}{l}\text { Prevenção de } \\
\text { situações de risco } \\
\text { a vida }\end{array}$ & $\begin{array}{l}\text { Risco de infecção (NANDA-I } \\
\text { 00004) (baseado em Nota } \\
\text { Técnica, ANVISA) }{ }^{26}\end{array}$ & $\begin{array}{l}\text { Realizar a higiene das mãos antes e após tocar o idoso para } \\
\text { os cuidados. } \\
\text { Disponibilizar álcool gel a 70\% para higiene das mãos nas } \\
\text { áreas comuns do domicílio e ILPI. } \\
\text { Auxiliar o idoso na higienização das mãos. } \\
\text { Manter uso de máscara enquanto abordar o idoso para } \\
\text { intervenções. } \\
\text { Supervisionar e realizar, quando necessário, a limpeza } \\
\text { e desinfecção de superfícies próximas do idoso e } \\
\text { frequentemente tocadas, bem como de equipamentos e } \\
\text { utensílios. } \\
\text { Orientar o idoso, familiares e/ou cuidadores para higiene } \\
\text { das mãos em atividades de cuidado, bem como manter o } \\
\text { uso de máscaras e a adoção da etiqueta da tosse e higiene } \\
\text { respiratória. } \\
\text { Orientar familiares e/ou cuidadores que habitualmente } \\
\text { estão circulando em locais susceptíveis de contaminação } \\
\text { por COVID19, que mantenham distanciamento mínimo de } \\
1 \text { metro do idoso. }\end{array}$ \\
\hline 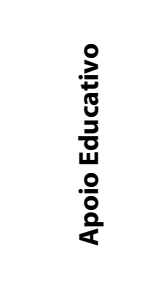 & 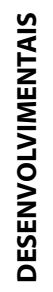 & $\begin{array}{l}\text { Provisão de } \\
\text { condições } \\
\text { promotoras de } \\
\text { desenvolvimento }\end{array}$ & $\begin{array}{l}\text { Interação social prejudicada } \\
\text { (NANDA-I 00052), condição } \\
\text { associada: isolamento } \\
\text { terapêutico (baseado na } \\
\text { intervenção "melhora do } \\
\text { sistema de apoio", NIC, 2016) }\end{array}$ & $\begin{array}{l}\text { Identificar o grau de apoio familiar, apoio financeiro } \\
\text { e recursos e rede de apoio disponíveis relacionados à } \\
\text { provisão de cuidados preventivos ao TEV, bem como as } \\
\text { medidas preventivas para COVID19. } \\
\text { Envolver a família, cuidadores e pessoas relevantes } \\
\text { (amigos) no planejamento de cuidados preventivos ao } \\
\text { TEV e COVID19, preferencialmente, utilizando recursos não } \\
\text { presenciais como ligação telefônica ou por videochamada. }\end{array}$ \\
\hline
\end{tabular}


Continuação do Quadro 1

\begin{tabular}{|c|c|c|c|c|}
\hline $\begin{array}{c}\text { Sistemas de } \\
\text { enfermagem }\end{array}$ & & $\begin{array}{l}\text { Requisitos de } \\
\text { autocuidado }\end{array}$ & $\begin{array}{c}\text { Diagnósticos de } \\
\text { enfermagem }\end{array}$ & Ações de enfermagem \\
\hline \multirow{5}{*}{ 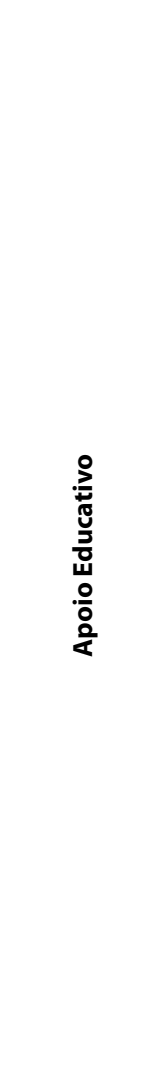 } & \multirow{2}{*}{ 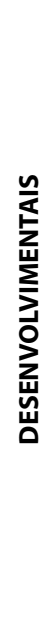 } & $\begin{array}{l}\text { Engajamento do } \\
\text { desenvolvimento } \\
\text { do eu }\end{array}$ & $\begin{array}{l}\text { Risco de baixa autoestima } \\
\text { situacional (NANDA-I 00153), } \\
\text { condição associada: prejuízo } \\
\text { funcional (baseado na } \\
\text { intervenção"controle do } \\
\text { humor, NIC, 2016) }\end{array}$ & $\begin{array}{l}\text { Incentivar o idoso a ter um papel ativo nas atividades } \\
\text { relacionadas a prevenção de eventos tromboembólicos, } \\
\text { bem como medidas preventivas a COVID19. } \\
\text { Monitorar o humor e funcionamento cognitivo do idoso, } \\
\text { utilizando instrumentos validados, preferencialmente. } \\
\text { Auxiliar o idoso a expressar seus sentimentos relacionados } \\
\text { a situação de distanciamento social. }\end{array}$ \\
\hline & & $\begin{array}{l}\text { Prevenção ou } \\
\text { superação }\end{array}$ & $\begin{array}{l}\text { Risco de sentimento de } \\
\text { impotência (NANDA-I } \\
\text { 00152), condição associada: } \\
\text { regime de tratamento } \\
\text { complexo (para prevenção } \\
\text { de TEV), baseado na } \\
\text { intervenção"facilitação da } \\
\text { aprendizagem", NIC, 2016) }\end{array}$ & $\begin{array}{l}\text { Proporcionar ambiente propício à aprendizagem, quando } \\
\text { no ensino as medidas preventivas de COVID19 e ao TEV, } \\
\text { incentivando a participação ativa do idoso. } \\
\text { Ajustar a instrução ao nível de conhecimento e } \\
\text { compreensão do idoso, utilizando linguagem familiar e } \\
\text { correspondências a objetos e/ou gestos. } \\
\text { Organizar as informações em uma sequência lógica, } \\
\text { considerando sessões curtas de ensino, conforme } \\
\text { apropriado e repetindo as informações importantes. } \\
\text { Fornecer feedback frequente sobre o progresso de } \\
\text { aprendizagem e reforçar os comportamentos preventivos } \\
\text { a COVID19 eTEV. }\end{array}$ \\
\hline & \multirow{3}{*}{ 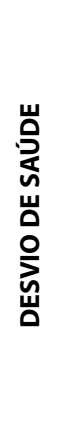 } & & $\begin{array}{l}\text { Orientar o idoso, familiares e/ou cuidadores quanto às medidas } \\
\text { preventivas para TEV (p. ex., evitar o tabagismo e consumo } \\
\text { excessivo de álcool; evitar longos períodos de imobilidade). }\end{array}$ \\
\hline & & \multirow{2}{*}{\multicolumn{2}{|c|}{$\begin{array}{l}\text { Risco de processo vascular prejudicado (CIPE } \\
\text { 10012993) e/ou } \\
\text { Risco de Embolia (CIPE 10051932) } \\
\text { Risco de perfusão tissular periférica ineficaz } \\
\text { (NANDA-I 00228) } \\
\text { Obter um histórico detalhado da saúde do idoso } \\
\text { a fim de determinar os fatores de risco para TEV e } \\
\text { apresentá-los. } \\
\text { Ensino ao idoso, familiar e/ou cuidador sobre os } \\
\text { sinais e sintomas de comprometimento venoso } \\
\text { periférico e embolia pulmonar. }\end{array}$}} & $\begin{array}{l}\text { Obter um histórico detalhado da saúde do idoso a fim de } \\
\text { determinar os fatores de risco para TEV e apresentá-los. }\end{array}$ \\
\hline & & & & $\begin{array}{l}\text { Ensino ao idoso, familiar e/ou cuidador sobre os sinais e } \\
\text { sintomas de comprometimento venoso periférico e embolia } \\
\text { pulmonar. }\end{array}$ \\
\hline \multirow{5}{*}{ 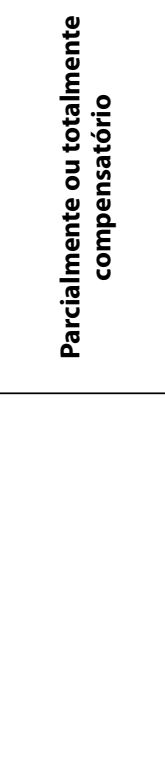 } & \multirow{3}{*}{ 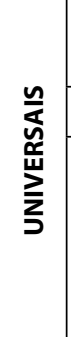 } & \multicolumn{2}{|c|}{ Volume de líquidos deficiente (NANDA-I 00027) } & \multirow{2}{*}{$\begin{array}{l}\text { Orientar familiares e/ou cuidadores quanto ao aumento da } \\
\text { oferta de líquidos ao idoso para garantir hidratação corporal. }\end{array}$} \\
\hline & & \multicolumn{2}{|c|}{ Ingestão de líquidos prejudicada (CIPE 10047330) } & \\
\hline & & \multicolumn{2}{|c|}{$\begin{array}{l}\text { Deambulação prejudicada (NANDA-I 00088) e/ou } \\
\text { Mobilidade Física prejudicada (NANDA-I 00085) }\end{array}$} & $\begin{array}{l}\text { Realizar o ensino aos familiares e/ou cuidadores sobre à } \\
\text { execução de exercícios ativos e passivos com amplitude do } \\
\text { movimento nos idosos. } \\
\text { Orientar sobre a importância da mudança de decúbito no } \\
\text { intervalo de duas em duas horas e de se evitar massagear ou } \\
\text { comprimir musculatura da perna. }\end{array}$ \\
\hline & \multicolumn{3}{|c|}{ DESENVOLVIMENTAIS } & Idem ações descritas no sistema apoio educativo. \\
\hline & 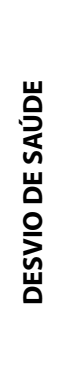 & \multicolumn{2}{|c|}{$\begin{array}{l}\text { Risco de confusão aguda (NANDA-I 00173) ou } \\
\text { Confusão crônica (NANDA-I 00129), condição } \\
\text { associada: demência (ações baseadas nas } \\
\text { intervenções e"orientação para a realidade"e } \\
\text { "musicoterapia" da NIC, 2016) }\end{array}$} & $\begin{array}{l}\text { Realizar abordagem de forma amistosa, lenta, para interação } \\
\text { com o (a) idoso (a), dirigindo-se pelo nome deste (a), e } \\
\text { envolvendo o familiar e/ou cuidador (quando apropriado). } \\
\text { Realizar uma pergunta e uma ordem/orientação de cada vez, } \\
\text { utilizando gestos, preferencialmente. } \\
\text { Modular os estímulos sensitivos humanos e ambientais de } \\
\text { forma a estimular o (a) idoso (a) memória e familiaridade com } \\
\text { o ambiente. } \\
\text { Utilizar música (audição passiva por oferta em caixas de som } \\
\text { amplificadoras, em cerca de } 30 \text { minutos) na modulação de } \\
\text { estímulo ambiental, buscando as preferências do (a) idoso (a), } \\
\text { observando o comportamento reativo desse (a). }\end{array}$ \\
\hline
\end{tabular}


Continuação do Quadro 1

\begin{tabular}{|c|c|c|c|c|}
\hline \multirow[t]{2}{*}{$\begin{array}{c}\text { Sistemas de } \\
\text { enfermagem }\end{array}$} & \multicolumn{2}{|c|}{$\begin{array}{c}\text { Requisitos de } \\
\text { autocuidado }\end{array}$} & $\begin{array}{l}\text { Diagnósticos de } \\
\text { enfermagem }\end{array}$ & Ações de enfermagem \\
\hline & 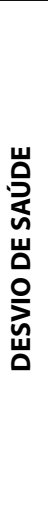 & \multicolumn{2}{|c|}{$\begin{array}{l}\text { Risco de processo vascular prejudicado (CIPE } \\
\text { 10012993) e/ou } \\
\text { Risco de Embolia (CIPE 10051932) } \\
\text { Risco de perfusão tissular periférica ineficaz } \\
\text { (NANDA-I 00228) }\end{array}$} & $\begin{array}{l}\text { Obter um histórico detalhado da saúde do idoso a fim de } \\
\text { determinar os fatores de risco para TEV e apresentá-los. } \\
\text { Ensinar ao idoso, familiar e/ou cuidador sobre os sinais e } \\
\text { sintomas de comprometimento venoso periférico e embolia } \\
\text { pulmonar. } \\
\text { Garantir correta administração de medicações profiláticas } \\
\text { conforme prescrição. } \\
\text { Elevar membro que se suponha estar afetado } 20^{\circ} \text { ou } \\
\text { mais acima do nível do coração para aumentar o retorno } \\
\text { venoso, observando os casos de cardiopatia e verificando } \\
\text { posicionamento que não desencadeie sobrecarga da coluna } \\
\text { lombar. } \\
\text { Aplicar meias antiembolia (p. ex., meias elásticas compressivas), } \\
\text { conforme apropriado. } \\
\text { Retirar as meias antiembolia por } 15 \text { ou } 20 \text { minutos, a cada oito } \\
\text { horas ou conforme indicado. }\end{array}$ \\
\hline
\end{tabular}

\section{Limitações do Estudo}

Como o tema é atual, ainda se dispõe de poucos estudos para ampliar a discussão, especialmente no que diz respeito aos idosos em risco de desenvolver eventos tromboembólicos em contextos extra-hospitalares, bem como, são necessários mais estudos que avaliem o impacto do distanciamento social e da infecção pelo vírus Sars-CoV-2 na ocorrência do TEV.

\section{Contribuições do ensaio reflexivo para a Enfermagem}

Uma das contribuições deste estudo para a área da enfermagem é a visibilidade da prevenção dos eventos tromboembólicos em idosos em distanciamento social, cujos cuidados podem ser negligenciados frente à emergência de outros recomendados no contexto da pandemia. Assim, essa reflexão contribui para a orientação e planejamento de estratégias no cuidado ao idoso.

Com essa reflexão evidencia-se também a necessidade da abordagem da enfermagem vascular no campo da enfermagem gerontológica, o que reforça a organização dessa práxis como especialidade numa perspectiva da integralidade do cuidado e sistematização da assistência ao idoso.

\section{CONSIDERAÇÕES FINAIS}

No atual panorama de pandemia da COVID-19, para garantir a proteção contra a infecção do novo Coronavírus, os idosos são orientados a permanecerem em seus lares. Porém, com essa medida de proteção, podem ser mais expostos aos fatores de risco para TEV. O enfermeiro deve buscar estratégias para minimizar barreiras que influenciem na aplicação das medidas de cuidado durante a situação pandêmica, garantindo acesso aos tratamentos e cuidados de saúde ao idoso.

\section{AGRADECIMENTO}

Ao Departamento Científico de Enfermagem Gerontológica da ABEn Nacional.

\section{REFERÊNCIAS}

1. Brooks, S. K., Webster, R. K., Smith, L. E., Woodland, L., Wessely, S., Greenberg, N., \& Rubin, G. J. (2020). The psychological impact of quarantine and how to reduce it: Rapid review of the evidence. The Lancet, 395(102227), 912-20. http://doi. org/10.1016/S0140- 6736(20)30460-8 
2. Yang, Y., Li, W., Zhang, Q., Zhang, L., Cheung, T., \& Xiang, Y.T. (2020). Mental health services for older adults in China during the COVID-19 outbreak. The Lancet Psychiatry, 7(e19). http://doi.org/101016/S2215-0366(20)30079-

3. Garcia LAA, Santos AS. A pandemia COVID-19 e as repercussões na atenção à saúde do idoso brasileiro. REFACS (online) Jul/Set 2020; 8(3):335-336. doi: 10.18554/refacs.v8i3.4557

4. Hammerschmidt KS de A, Santana RF. Saúde do idoso em tempos de pandemia Covid-19. Cogitare enferm. [Internet]. 2020 [acesso em 19 jun 2020]; 25. Disponível em: http:// dx.doi.org/10.5380/ce.v25i0.72849.

5. Constandt B, Thibau E, Bosscher V, Scheerder J, Ricour M, Willem A. Exercising in Times of Lockdown: An Analysis of the Impact of COVID-19 on Levels and Patterns of Exercise among Adults in Belgium. Int. J. Environ. Res. Public Health 2020, 17(11), 4144; https://doi.org/10.3390/ijerph17114144

6. Goodman-Casanova JM, Dura-Perez E, Guzman-Parra J, Cuesta-Vargas A, Mayoral-Cleries F. Telehealth Home Support During COVID-19 Confinement for Community-Dwelling Older Adults With Mild Cognitive Impairment or Mild Dementia: Survey Study. J Med Internet Res. 2020;22(5):e19434. Published 2020 May 22. doi:10.2196/19434.

7. Phillippe HM. Overview of venous thromboembolism. Am J Manag Care. 2017;23(20 Suppl):S376-S382. Available from: https://ajmc.s3.amazonaws.com/_media/_pdf/Overview\%20of\%20Venous\%20Thromboembolism.pdf

8. Tana C, Lauretani F, Ticinesi A, Prati B, Nouvenne A, Meschi T. Molecular and Clinical Issues about the Risk of Venous Thromboembolism in Older Patients: A Focus on Parkinson's Disease and Parkinsonism. Int J Mol Sci. 2018;19(5):1299. Published 2018 Apr 26. doi:10.3390/ijms19051299.

9. Zhang Zhu, Lei Jieping, Shao Xiang, Dong Fen, Wang Jing, Wang Dingyi et al. Trends in Hospitalization and In-Hospital Mortality From VTE, 2007 to 2016, in China. Chest. 2019; 155(2): 342-353. doi: https://doi.org/10.1016/j.chest.2018.10.040

10. Apenteng PN, Hobbs FR, Roalfe A, Muhammad U, Heneghan C, Fitzmaurice D. Incidence of venous thromboembolism in care homes: a prospective cohort study. Br J Gen Pract. 2017;67(655):e130-e137. doi:10.3399/bjgp17X688873

11. Ahmed J, Ornstein K, Dunn A, Gliatto P et al. Incidence of Venous Thromboembolism in a Homebound Population. $J$ Community Health. 2013;38:480-485. doi: https://doi.org/10.1007/s10900-012-9632-2

12. Münster AM, Rasmussen TB, Falstie-Jensen AM, Harboe L, Stynes G, Dybro L et al. A changing landscape: Temporal trends in incidence and characteristics of patients hospitalized with venous thromboembolism 2006-2015. Thromb Res.. 2019;176:46-53. doi:10.1016/j.thromres.2019.02.009

13. Lauber Sandro, Limacher Andreas, Tritschler Tobias, Stalder Odile, Méan Marie, Righini Marc et al. Predictors and Outcomes of Recurrent Venous Thromboembolism in Elderly Patients. Am J Med. 2018; 131(6):703.e7-703.e16. doi: https://doi. org/10.1016/j.amjmed.2017.12.015

14. Xia ZN, Xiao K, Zhu W, Feng B, Zhang BZ, Lin J et al. Risk assessment and management of preoperative venous thromboembolism following femoral neck fracture. J Orthop Surg Res. 2018;13(1):291. doi: 10.1186/s13018-018-0998-4

15. Arpaia G, Ambrogi F, Penza M, lanes AB, Serras A, Boracchi P, et al. Risk of venous thromboembolism in patients nursed at home or in long-term care residential facilities. Int J Vasc Med. 2011;2011.doi: 10.1155/2011/305027

16. Bjøri E, Johnsen HS, Hansen JB, Brækkan SK. Hospitalization as a trigger for venous thromboembolism - Results from a population-based case-crossover study. Thromb Res. 2019; 176:115-119. doi:10.1016/j.thromres.2019.02.024

17. Folsom AR, Boland LL, Cushman M, Heckbert SR, Rosamond WD, Walston JD. Frailty and risk of venous thromboembolism in older adults. J Gerontol A Biol Sci Med Sci. 2007;62(1):79-82. doi:10.1093/gerona/62.1.79

18. Zhai Z, Li C, Chen Y, et al. Prevention and Treatment of Venous Thromboembolism Associated with Coronavirus Disease 2019 Infection: A Consensus Statement before Guidelines. Thromb Haemost. 2020;120(6):937-948. doi:10.1055/s-0040-1710019

19. Orem DE, Taylor SG, Renpenning KM. Nursing Concepts of practice. 5 ed. St. Louis Missouri: Mosby; 1995.

20. Taylor SG, Renpenning K. Self-Care Sciences, Nursing Theory, and Evidence-Based Practice. New York: Springer Publishing Company; 2011. $101 \mathrm{p}$.

21. Evensen LH, Isaksen T, Braekkan SK, Hansen JB. Cardiorespiratory fitness and future risk of venous thromboembolism. J Thromb Haemost. 2019;17(12):2160-2168. doi:10.1111/jth.14619

22. Bulecheck GM, Butcher HK, Dochterman JM, Wagner CM. Classificação das Intervenções de Enfermagem. Rio de Janeiro: Elsevier; 2016. 640 p. 
23. NANDA-I. Diagnósticos de Enfermagem da NANDA-I: definições e classificações: 2018-2020. 11a ed. Porto Alegre: Artmed; 2018. $488 \mathrm{p}$.

24. Garcia TR. CIPE ${ }^{\oplus}$ Classificação Internacional para Prática de Enfermagem: Versão 2019-2020. $1^{\text {a }}$ ed. Porto Alegre: Artmed; 2019. 352 p.

25. Nunes $C A B$, Melo PG, Malaquias SG, et al. Effectiveness of two bundles in venous leg ulcer healing: a randomized controlled trial. Journal of Vascular. 2019;37(4):232-245. doi: 10.1016/j.jvn.2019.09.004

26. Agência Nacional de Vigilância Sanitária. Gerência Geral de Tecnologia em Serviços de Saúde. Gerência de Vigilância e Monitoramento em Serviços de Saúde. Orientações para a prevenção e o controle de infecções pelo novo coronavírus (SARS-CoV-2) em instituições de longa permanência para idosos (ILPI). Nota Técnica GVIMS/GGTES/ANVISA nº 05/2020. Disponível em: file:///C:/Users/milar/Downloads/NOTA_TCNICA_05_2020_GVIMS_GGTES.pdf 